\title{
HARMONIC ANALYSIS AND ULTRACONTRACTIVITY
}

\author{
MICHAEL COWLING AND STEFANO MEDA
}

\begin{abstract}
Let $\left(T_{t}\right)_{t>0}$ be a symmetric contraction semigroup on the spaces $L^{p}(M) \quad(1 \leq p \leq \infty)$, and let the functions $\phi$ and $\psi$ be "regularly related". We show that $\left(T_{t}\right)_{t>0}$ is $\phi$-ultracontractive, i.e., that $\left(T_{t}\right)_{t>0}$ satisfies the condition $\left\|T_{t} f\right\|_{\infty} \leq C \phi(t)^{-1}\|f\|_{1}$ for all $f$ in $L^{1}(M)$ and all $t$ in $\mathbf{R}^{+}$, if and only if the infinitesimal generator $\mathscr{G}$ has Sobolev embedding properties, namely, $\left\|\psi(\mathscr{G})^{-\alpha} f\right\|_{q} \leq C\|f\|_{p}$ for all $f$ in $L^{p}(M)$, whenever $1<p<q<\infty$ and $\alpha=1 / p-1 / q$. We establish some new spectral multiplier theorems and maximal function estimates. In particular, we give sufficient conditions on $m$ for $m(\mathscr{G})$ to map $L^{p}(M)$ to $L^{q}(M)$, and for the example where there exists $\mu$ in $\mathbf{R}^{+}$such that $\phi(t)=t^{\mu}$ for all $t$ in $\mathbf{R}^{+}$, we give conditions which ensure that the maximal function $\sup _{t>0}\left|t^{\alpha} T_{t} f(\cdot)\right|$ is bounded.
\end{abstract}

Let $\mathscr{G}$ be a positive, possibly unbounded, but with dense domain, operator on $L^{2}(M)$, where $M$ is a $\sigma$-finite measure space. Let $\left\{P_{\lambda}\right\}$ be the spectral resolution of the identity for which

$$
\mathscr{G} f=\int_{0}^{\infty} \lambda d P_{\lambda} f \quad \forall f \in \operatorname{Dom}(\mathscr{G}) .
$$

For every positive real number $t$, we define the operator $T_{t}$ by the rule

$$
T_{t} f=\int_{0}^{\infty} e^{-t \lambda} d P_{\lambda} f \quad \forall f \in L^{2}(M) .
$$

We assume throughout that each $T_{t}$ has the contraction property

$$
\left\|T_{t} f\right\|_{p} \leq\|f\|_{p} \quad \forall f \in L^{2}(M) \cap L^{p}(M)
$$

whenever $1 \leq p \leq \infty$. We also assume for convenience that the spectral projection $P_{0}$ is trivial on $L^{p}(M)$ whenever $1 \leq p<\infty$. The theory can readily be modified to take into account the possibility that $P_{0}$ is nontrivial, but the statements become more complicated; we find the extra generality does not merit the extra complexity. A semigroup $\left(T_{t}\right)_{t>0}$ with the above properties will be called a symmetric contraction semigroup.

A symmetric contraction semigroup $\left(T_{t}\right)_{t>0}$ for which $T_{t}$ maps $L^{1}(M)$ into $L^{\infty}(M)$ for every positive $t$ is said to be ultracontractive. The notion of ultracontractivity appeared compatively recently in work of E. B. Davies [4] and Davies and B. Simon [6], in connection with logarithmic Sobolev inequalities

Received by the editors October 3, 1990 and in revised form, September 30, 1991.

1991 Mathematics Subject Classification. Primary 47D05.

Research supported by the Australian Research Council and the Italian Ministero della Pubblica Istruzione. 
(see also L. Gross [7]). N. T. Varopoulos [16] developed a Hardy-Littlewood theory for semigroups. In particular he proved, under stronger assumptions on the semigroup $\left(T_{t}\right)_{t>0}$ and on the underlying measure space $M$, that an estimate of the form

$$
\left\|T_{t} f\right\|_{\infty} \leq A t^{-\mu}\|f\|_{1} \quad \forall t \in \mathbf{R}^{+}, \forall f \in L^{1}(M)
$$

for some $\mu$ greater than 1 is equivalent to the boundedness of the "Riesz potential operator" $\mathscr{G}^{-1 / 2}$ from $L^{2}(M)$ to $L^{q}(M)$, where $1 / 2-1 / q=1 /(2 \mu)$. Varopoulos proved the hard part of this result (namely, that the boundedness of $\mathscr{G}^{-1 / 2}$ implies ultracontractivity) using an extension to the abstract semigroup setting of the iterative process of $J$. Moser $[10,11]$ for parabolic equations. Davies [5, Theorem 2.4.2] gave a different proof which relies on the relationship between logarithmic Sobolev inequalities and ultracontractivity. Other simplifications have been discovered by E. A. Carlen, S. Kusuoka, and D. W. Stroock [1], who related ultracontractivity to Nash inequalities, and by T. Coulhon [2], who also formulated versions of the equivalences for the case when

$$
\left\|T_{t} f\right\|_{\infty} \leq C_{0} t^{-\mu}\|f\|_{1} \quad \forall t \in(0,1], \forall f \in L^{1}(M),
$$

and

$$
\left\|T_{t} f\right\|_{\infty} \leq C_{1} t^{-\nu}\|f\|_{1} \quad \forall t \in[1, \infty), \forall f \in L^{1}(M),
$$

where $\mu$ and $\nu$ are positive.

By the closed graph theorem, every ultracontractive semigroup satisfies an estimate of the form

$$
\left\|T_{t} f\right\|_{\infty} \leq A \phi(t)^{-1}\|f\|_{1} \quad \forall t \in \mathbf{R}^{+}, \forall f \in L^{1}(M),
$$

for some increasing function $\phi: \mathbf{R}^{+} \rightarrow \mathbf{R}^{+}$, and $A$ equal to 1 . For a fixed function $\phi$, the semigroups which satisfy $(0.2)$ (for any $A$ in $\mathbf{R}^{+}$) will be called $\phi$-ultracontractive. Note that multiplying the measure on the underlying measure space by a constant factor amounts to varying $A$; the definition of $\phi$-ultracontractivity is thus independent of such a change of measure. Davies' work [5] concerns these general semigroups. It is interesting to speculate how such more general ultracontractive estimates may be related to inequalities of Sobolev type. Examples such as heat diffusions on hyperbolic spaces suggest that exponential growth of $\phi$ is related to "spectral gap" phenomena, and rather different sorts of results are to be expected from those in the classic example of heat diffusion in Euclidean space, when $\phi$ grows polynomially.

In this paper, we present a version of the equivalence of various Sobolev immersions and ultracontractivity, under hypotheses which include the polynomial growth of $\phi$, which clarifies the underlying function theory. Our results are more general than those of the authors mentioned above; in particular, we can deal with more general $\phi$ than the previous workers (except Davies, and our proofs are simpler than his, as we do not require that $T_{t}$ preserve positivity for all $t$ in $\mathbf{R}^{+}$, nor do we use logarithmic Sobolev inequalities), and we include in our equivalences a weak-type Sobolev embedding which has apparently not been noted before.

Let $m$ be a complex-valued, Borel measurable function on $\mathbf{R}^{+} \cup\{0\}$, and take $t$ in $\mathbf{R}^{+}$. The "multiplier operator" $m(t \mathscr{G})$ is then defined on $L^{2}(M)$ by the rule $m(t \mathscr{G}) f=\int_{0}^{\infty} m(t \lambda) d P_{\lambda} f$, whenever $f$ is in the appropriate domain. 
In particular, we denote the operator $m(1 \mathscr{G})$ by $m(\mathscr{G})$. We develop a functional calculus for infinitesimal generators of symmetric contraction semigroups satisfying (0.2). We prove that under suitable conditions of "Hörmander type" on the function $m$, the operator $m(\mathscr{G})$ is bounded from $L^{p}(M)$ to $L^{q}(M)$, when $1<p \leq q<\infty$. (More precisely, $m(\mathscr{G})$ maps $L^{p}(M) \cap \operatorname{Dom}(m(\mathscr{G})$ ) into $L^{q}(M)$, and extends uniquely to a continuous map from $L^{p}(M)$ to $L^{q}(M)$.) We complement and develop the theory of E. M. Stein [13], Cowling [3] and Meda [9], who examined the case where $p=q$.

For any nonnegative $\alpha$ we may define, at least formally, the maximal operator $m_{*, \alpha}(\mathscr{G})$ associated to the family $(m(t \mathscr{G}))_{t>0}$ by the formula

$$
m_{*, \alpha}(\mathscr{G}) f=\sup _{t>0}\left|t^{\alpha} m(t \mathscr{G}) f\right| .
$$

In the case where $m(\lambda)=\exp (-\lambda)$, we write $T_{*, \alpha}$ instead of $m_{*, \alpha}(\mathscr{G})$. One of the main results of this paper is that, for a symmetric contraction semigroup satisfying condition (0.1) above, the maximal operator $T_{*, \alpha}$ is of weak type $(1, r)$, when $1<r \leq \infty$ and $1-1 / r=\alpha / \mu$.

We shall present our results in four sections. In the first, we consider ultracontractivity, and relate this to estimates for the resolvent of $\mathscr{G}$. We show that, when $\phi$ and $\psi$ are "regularly related", an estimate of the form $(0.2)$ is equivalent to one of the form

$$
\left\|T_{t} f\right\|_{q} \leq A \phi(t)^{-\alpha}\|f\|_{p} \quad \forall t \in \mathbf{R}^{+}, \forall f \in L^{p}(M)
$$

for some or all $p, q$, and $\alpha$ such that $1 \leq p<q \leq \infty$ and $\alpha=1 / p-1 / q$, and is also equivalent to ones of the form

$$
\left\|\psi(\mathscr{G})^{-\alpha} f\right\|_{q} \leq B\|f\|_{p} \quad \forall f \in L^{p}(M)
$$

or

$$
\left\|\psi(\mathscr{G})^{-\alpha} f\right\|_{q, \infty} \leq B\|f\|_{p} \quad \forall f \in L^{p}(M),
$$

for some or all $p, q$, and $\alpha$ such that $1<p<q<\infty$ and $\alpha=1 / p-1 / q$.

In $\S 2$, we examine multiplier results for $\phi$-ultracontractive semigroups. With the additional hypothesis (often verified) that there is a positive number $\sigma$ such that

$$
\left\|\mathscr{G}^{i u} f\right\|_{p} \leq C_{p}(1+|u|)^{\sigma|1 / p-1 / 2|}\|f\|_{p} \quad \forall u \in \mathbf{R}, \forall f \in L^{p}(M),
$$

we prove Hörmander-like multiplier theorems: if $\phi$ and $\psi$ are regularly related, and $m: \mathbf{R}^{+} \rightarrow \mathbf{C}$ satisfies the hypothesis that

$$
\left|\lambda^{k}(\partial / \partial \lambda)^{k} m(\lambda)\right| \leq C \psi(\lambda)^{-\alpha} \quad \forall \lambda \in \mathbf{R}^{+}, \forall k \in\{0,1, \ldots, \chi\}
$$

where $\chi$ is an integer no less than $\sigma / 2+1$, then $m(\mathscr{G})$ is of weak type $(1, r)$ and of strong type $(p, q)$, when $1<r<\infty, 1<p<q<\infty$ and $\alpha=1-1 / r=$ $1 / p-1 / q$. These results generalize work of the second-named author [9], who proved Hörmander-type theorems for symmetric contraction semigroups.

In $\S 3$, we establish various results about maximal functions. In particular, we show that for $\mu$-ultracontractive semigroups, $T_{*, \alpha}$ is of weak type $(1, r)$ when $1<r \leq \infty$ and $1-1 / r=\alpha / \mu$, i.e.,

$$
\left\|\sup _{t>0}\left|t^{\alpha} T_{t} f(\cdot)\right|\right\|_{r, \infty} \leq C\|f\|_{1} \quad \forall f \in L^{1}(M) .
$$


Further, $T_{*, \alpha}$ is of strong type $(p, q)$ when $1<p<q<\infty$ and $1 / p-1 / q=$ $\alpha / \mu$.

In $\S 4$, we consider generalizations of the preceding results. There are interesting semigroups for which the appropriate ultracontractivity estimate is the following:

$$
\left\|T_{t} f\right\|_{\infty} \leq A t^{-\mu}\|f\|_{1} \quad \forall t \in(0,1], \forall f \in L^{1}(M) .
$$

We give versions of our results for these semigroups.

A little notation might be in order. The usual Lebesgue and Lorentz spaces on the measure space $M$ are denoted $L^{p}(M)$ and $L^{p, q}(M)$ respectively. A convenient reference for these is the book by Stein and G. Weiss [14]. The norm of a bounded linear operator $T$ on one of these spaces will be denoted by $\||| T\|_{p}$ or by $\||T|\|_{p, q}$ respectively. An operator which is bounded from $L^{p}(M)$ to $L^{q}(M)$ or from $L^{p}(M)$ to $L^{q, \infty}(M)$ is said to be of strong type $(p, q)$ or of weak type $(p, q)$ respectively. The corresponding norms are denoted by ||$T \mid \|_{p ; q}$ and ||$T \mid \|_{p ; q, \infty}$. We denote the sector $\{z \in \mathbf{C}:|\arg (z)|<\omega\}$ by $\Gamma_{\omega}$, the halfplane $\Gamma_{\pi / 2}$ by $\Gamma$, and the space of bounded holomorphic functions on the domain $\Omega$ by $H^{\infty}(\Omega)$. Constants will be denoted with the letters $A, B$, and $C$, sometimes with subscripts. The same symbol may be used to denote different constants.

We conclude our introduction by recalling one of the principal results of Cowling [3]. Let $\left(T_{t}\right)_{t>0}$ be a symmetric contraction semigroup on a $\sigma$-finite measure space $M$, with generator $\mathscr{G}$, and suppose that $1<p<\infty$. If $\omega\rangle$ $\pi|1 / p-1 / 2|$, and $m$ extends to a $H^{\infty}\left(\Gamma_{\omega}\right)$-function, denoted $m_{\omega}$, then $m(\mathscr{G})$ is of strong type $(p, p)$, and

$$
\|\| m(\mathscr{G})\|\|_{p} \leq C_{p, \omega}\left\|m_{\omega}\right\|_{\infty} .
$$

By applying the real interpolation method, we may easily deduce that $m(\mathscr{G})$ is also bounded on $L^{q, \infty}(M)$ whenever $q$ lies between 2 and $p$, and that

$$
\|\| m(\mathscr{G})\left\|_{q, \infty} \leq C_{q, \omega}^{\prime}\right\| m_{\omega} \|_{\infty} .
$$

In particular it follows that for every real number $u$ the operator $\mathscr{G}^{i u}$ is bounded on $L^{p}(M)$ and on $L^{p, \infty}(M)$ whenever $1<p<\infty$, and that for all positive $\delta$,

$$
|| \mathscr{G}^{i u} \mid \|_{p} \leq C_{p, \delta} \exp ((\pi|1 / p-1 / 2|+\delta)|u|) \quad \forall u \in \mathbf{R},
$$

and

$$
\left\|\left|\mathscr{G}^{i u}\right|\right\|_{p, \infty} \leq C_{p, \delta}^{\prime} \exp ((\pi|1 / p-1 / 2|+\delta)|u|) \quad \forall u \in \mathbf{R} .
$$

This is an easy improvement of a result of Stein [13, Corollary 4, p. 121].

This paper was begun while the first author was visiting the Università di Trento, and completed while the second author was visiting the University of New South Wales. Both authors wish to express their gratitude to the host institutions for their kind hospitality. The referee of the first version of this paper brought some recent papers to our attention and thereby stimlated some improvements; we thank him or her for these references.

\section{ULTRACONTRACTIVITY AND RESOLVENT ESTIMATES}

A pair of functions $\phi: \mathbf{R}^{+} \rightarrow \mathbf{R}^{+}$and $\psi: \Gamma \rightarrow \mathbf{C}$ will be called "regularly related" if 
(a) $\phi$ is increasing, continuous, and surjective;

(b) there exists a constant $C_{\phi}$ such that

$$
\phi(2 t) \leq C_{\phi} \phi(t) \quad \forall t \in \mathbf{R}^{+} ;
$$

(c) for all $\theta$ in $\mathbf{R}^{+}$, there exists a constant $D_{\phi, \theta}$ such that

$$
\int_{0}^{t} \phi(s)^{\theta} \frac{d s}{s} \leq D_{\phi, \theta} \phi(t)^{\theta} \quad \forall t \in \mathbf{R}^{+}
$$

and

$$
\int_{t}^{\infty} \phi(s)^{-\theta} \frac{d s}{s} \leq D_{\phi, \theta} \phi(t)^{-\theta} \quad \forall t \in \mathbf{R}^{+}
$$

(d) $\psi$ is holomorphic in $\Gamma$, and $\psi(\bar{z})=\overline{\psi(z)}$ for all $z$ in $\Gamma$;

(e) for all $\omega$ in $(0, \pi / 2)$, there exists a constant $C_{\psi, \omega}$ such that

$$
\inf \left\{|\psi(z)|: z \in S_{R, \omega}\right\} \geq C_{\psi, \omega} \sup \left\{|\psi(z)|: z \in S_{R, \omega}\right\}, \quad \forall R \in \mathbf{R}^{+}
$$

where $S_{R, \omega}=\{z \in \mathbf{C}: R<\operatorname{Re}(z)<2 R,|\arg (z)|<\omega\}$;

(f) for all $\theta$ in $\mathbf{R}^{+}$, there exists a real-valued measurable function $\phi_{\theta}$, equivalent to $\phi^{\theta}$ in the sense that the function $t \mapsto\left|\phi(t)^{\theta} / \phi_{\theta}(t)\right|$ is bounded and bounded away from 0 in $\mathbf{R}^{+}$, with the property that, if

$$
\psi_{\theta}(z)=\int_{0}^{\infty} e^{-z t} \phi_{\theta}(t) \frac{d t}{t} \quad \forall z \in \Gamma,
$$

then $\psi_{\theta}$ is equivalent to $\psi^{-\theta}$ in the sense that the function $z \mapsto\left|\psi(z)^{\theta} \psi_{\theta}(z)\right|$ is bounded and bounded away from 0 in any proper subcone $\Gamma_{\omega}$ of $\Gamma$.

One obvious example of a pair of regularly related functions is when $\phi(t)=t^{\mu}$ and $\psi(z)=z^{\mu}$ for some positive $\mu$; in this case $\phi_{\theta}$ may be taken to be $\phi^{\theta}$. The key to understanding this example is the integral formula

$$
\int_{0}^{\infty} t^{\theta \mu} e^{-z t} \frac{d t}{t}=\Gamma(\theta \mu) z^{-\theta \mu} \quad \forall z \in \Gamma .
$$

Less trivial examples are given in the following lemma.

Lemma 1.1. (a) Suppose that $\mu, \nu \in \mathbf{R}^{+}$, that $\phi(t)=t^{\mu}(1+t)^{\nu-\mu}$ for all $t$ in $\mathbf{R}^{+}$, and that $\psi(z)=z^{\nu}(1+z)^{\mu-\nu}$ for all $z$ in $\Gamma$. Then $(\phi, \psi)$ is a regularly related pair.

(b) Suppose that $\mu, \nu \in \mathbf{R}^{+}$, that $\phi(t)=t^{\mu} \log (2+t)^{\nu}$ for all $t$ in $\mathbf{R}^{+}$, and that $\psi(z)=z^{\mu} \log (2+1 / z)^{-\nu}$ for all $z$ in $\Gamma$. Then $(\phi, \psi)$ is a regularly related pair.

Proof. To prove (a), one first verifies that $\phi$ and $\psi$ satisfy conditions (a) to (e); this is routine. The function $\phi_{\theta}$ in (f) is given by the formula

$$
\phi_{\theta}(t)=\frac{t}{\Gamma(\theta(\mu-\nu)) \Gamma(\theta \nu)} \int_{0}^{t} s^{\theta \nu-1}(t-s)^{\theta(\mu-\nu)-1} \exp (s-t) d s \quad \forall t \in \mathbf{R}^{+},
$$

which is a well-defined integral when $\mu>\nu$ and is interpreted by analytic continuation for other values of $\mu$. Indeed, when $\mu>\nu$, by changing variables (putting $s=t(1-\tau)$ ) and writing the exponential as a series, we see that, for 
any $t$ in $\mathbf{R}^{+}$,

$$
\begin{aligned}
\phi_{\theta}(t) & =\frac{t^{\theta \mu}}{\Gamma(\theta(\mu-\nu)) \Gamma(\theta \nu)} \int_{0}^{1} \tau^{\theta(\mu-\nu)-1}(1-\tau)^{\theta \nu-1} \exp (-t \tau) d \tau \\
& =\sum_{n=0}^{\infty}(-t)^{n} t^{\theta \mu} \frac{\Gamma(\theta(\mu-\nu)+n)}{\Gamma(\theta(\mu-\nu)) \Gamma(\theta \mu+n) n !},
\end{aligned}
$$

which continues analytically in $\mu$ into the whole complex plane; further, for small $t, \phi_{\theta}(t)$ behaves like $t^{\mu \theta}$. Moreover, by changing variables again (putting $\sigma=t \tau)$, we see that

$$
\phi_{\theta}(t)=\frac{t^{\nu \theta}}{\Gamma(\theta(\mu-\nu)) \Gamma(\theta \nu)} \int_{0}^{t}\left(1-\frac{\sigma}{t}\right)^{\theta \nu-1} \exp (-\sigma) \sigma^{\theta(\mu-\nu)-1} d \sigma .
$$

If $t>1$, we may write the integral as a sum of an integral between 0 and 1 and one between 1 and $t$. The first of these (with the term $t^{\nu \theta}$ ) expands as a convergent series of the form $\sum_{n=0}^{\infty} a_{n} t^{\nu \theta-n}$, in which $a_{0}>0$, while Lebesgue's dominated convergence theorem shows that the second integral (without the term $t^{\nu \theta}$ ) tends to $\int_{1}^{\infty} \exp (-\sigma) \sigma^{\theta(\mu-\nu)-1} d \sigma$. Consequently, $\phi_{\theta}$ is equivalent to $\phi^{\theta}$. By using the properties of the Laplace transform, it is easy to show that $\psi_{\theta}=\psi^{-\theta}$, concluding the proof of (a).

To prove (b), it again suffices to consider the last condition only, as the others hold trivially. The function $\phi_{\theta}$ is taken to be $t \mapsto t^{\theta \mu} \log (K+t)^{\theta \nu}$, for a sufficiently large value of $K$ (whose size will be explained later). Then, if $r$ is in $\mathbf{R}^{+}$and $\omega$ is in $(-\pi / 2, \pi / 2)$,

$$
\begin{aligned}
\psi_{\theta}\left(r e^{i \omega}\right) & =\int_{0}^{\infty} e^{-r e^{i \omega} t} t^{\theta \mu-1} \log (K+t)^{\theta \nu} d t \\
& =e^{-i \omega \theta \mu} \int_{0}^{\infty} e^{-r t} t^{\theta \mu-1} \log \left(K+t e^{-i \omega}\right)^{\theta \nu} d t,
\end{aligned}
$$

by a contour integration argument, and so

$$
e^{i \omega \theta \mu} \psi_{\theta}\left(r e^{i \omega}\right)=\int_{0}^{\infty} e^{-r t} t^{\theta \mu-1} \log \left(K+t e^{-i \omega}\right)^{\theta \nu} d t
$$

If $K$ is so large that $\log \left(K+t e^{-i \omega}\right)$ has argument of absolute value less than $\pi / 6(1+|\theta \nu|)$, for all $t$ in $\mathbf{R}^{+}$, then the argument of the integrand lies sufficiently close to 0 that the real part of the integrand is always positive and at least one half of the absolute value. Then it follows that

$$
\begin{aligned}
\int_{0}^{\infty} & e^{-r t} t^{\theta \mu-1} \operatorname{Re}\left(\log \left(K+t e^{-i \omega}\right)^{\theta \nu}\right) d t \\
& =\operatorname{Re}\left(e^{i \omega \theta \mu} \psi_{\theta}\left(r e^{i \omega}\right)\right) \leq\left|\psi_{\theta}\left(r e^{i \omega}\right)\right| \\
& \leq \int_{0}^{\infty} e^{-r t} t^{\theta \mu-1}\left|\log \left(K+t e^{-i \omega}\right)^{\theta \nu}\right| d t \\
& \leq 2 \int_{0}^{\infty} e^{-r t} t^{\theta \mu-1} \operatorname{Re}\left(\log \left(K+t e^{-i \omega}\right)^{\theta \nu}\right) d t
\end{aligned}
$$

i.e., that $\psi_{\theta}\left(r e^{i \omega}\right)$ is comparable to $\int_{0}^{\infty} e^{-r t} t^{\theta \mu-1}\left|\log \left(K+t e^{-i \omega}\right)^{\theta \nu}\right| d t$, which in turn is easily seen to be comparable to $\int_{0}^{\infty} e^{-r t} t^{\theta \mu-1} \log (K+t)^{\theta \nu} d t$ and then to $\psi\left(r e^{i \omega}\right)^{-\theta}$, as required. 
It might well be possible, and worthwhile, to find simpler function theoretic criteria for describing such pairs.

Here is our first main theorem.

Theorem 1.2. Let $\phi$ and $\psi$ be a pair of regularly related functions, and let $\left(T_{t}\right)_{t>0}$ be a symmetric contraction semigroup. The following conditions are equivalent:

$$
\left\|T_{t} f\right\|_{\infty} \leq A \phi(t)^{-1}\|f\|_{1} \quad \forall t \in \mathbf{R}^{+}, \forall f \in L^{1}(M) ;
$$

(b) for all $p, q$ and $\alpha$ such that $1 \leq p<q \leq \infty$, and $\alpha=1 / p-1 / q$,

$$
\left\|T_{t} f\right\|_{q} \leq A^{\prime} \phi(t)^{-\alpha}\|f\|_{p} \quad \forall t \in \mathbf{R}^{+}, \forall f \in L^{p}(M) ;
$$

(c) there exist $p, q$ and $\alpha$ such that $1 \leq p<q \leq \infty, \alpha=1 / p-1 / q$, and

$$
\left\|T_{t} f\right\|_{q} \leq A^{\prime} \phi(t)^{-\alpha}\|f\|_{p} \quad \forall t \in \mathbf{R}^{+}, \forall f \in L^{p}(M) ;
$$

(d) for all $r$ and $\alpha$ such that $1<r<\infty$, and $\alpha=1-1 / r$,

$$
\left\|\psi(\mathscr{G})^{-\alpha} f\right\|_{r, \infty} \leq C_{r}\|f\|_{1} \quad \forall f \in L^{1}(M) ;
$$

(e) for all $p, q$ and $\alpha$ such that $1<p<q<\infty$, and $\alpha=1 / p-1 / q$,

$$
\left\|\psi(\mathscr{G})^{-\alpha} f\right\|_{q} \leq C_{p, q}\|f\|_{p} \quad \forall f \in L^{p}(M) ;
$$

(f) there exist $p, q$ and $\alpha$ such that $1<p<q<\infty, \alpha=1 / p-1 / q$, and

$$
\left\|\psi(\mathscr{G})^{-\alpha} f\right\|_{q} \leq C\|f\|_{p} \quad \forall f \in L^{p}(M) ;
$$

(g) there exist $p, q$ and $\alpha$ such that $1<p<q<\infty, \alpha=1 / p-1 / q$, and

$$
\left\|\psi(\mathscr{G})^{-\alpha} f\right\|_{q, \infty} \leq C\|f\|_{p} \quad \forall f \in L^{p}(M) .
$$

Remark. Note that we are assuming in (a) that $A \phi(t)^{-1} \geq\left\|T_{t}\right\|_{1 ; \infty}$ for all $t$ in $\mathbf{R}^{+}$, but not that equality holds. Similar comments apply about (b) and (c).

Proof. By interpolating between the estimates

$$
\left\|T_{t} f\right\|_{r} \leq\|f\|_{r} \quad \forall t \in \mathbf{R}^{+}, \forall f \in L^{r}(M)
$$

and

$$
\left\|T_{t} f\right\|_{\infty} \leq \phi(t)^{-1}\|f\|_{1} \quad \forall t \in \mathbf{R}^{+}, \forall f \in L^{1}(M),
$$

using the complex interpolation method, it is easy to show that (a) implies (b). Similarly, by using the real interpolation method between (d) and its dual estimate, namely

$$
\left\|\psi(\mathscr{G})^{-\alpha} f\right\|_{\infty} \leq C_{r}\|f\|_{r^{\prime}, 1} \quad \forall f \in L^{r^{\prime}, 1}(M),
$$

it can be shown that (d) implies (e), and that the constant in (e) depends linearly on that in (d). Trivially, (b) implies (c), (e) implies (f), and (f) implies (g). We shall complete our proof by showing that (c) implies (a), (a) implies (d), and (g) implies (c). These three implications will be entitled Lemma 1.3, Lemma 1.4 and Lemma 1.5 respectively. Lemma 1.3 is a generalization of a result of T. Coulhon [2]. 
Lemma 1.3. Suppose that $\left(T_{t}\right)_{t>0}$ is a symmetric contraction semigroup satisfying the condition

$$
\left\|T_{t} f\right\|_{q} \leq \phi(t)^{-\alpha}\|f\|_{p} \quad \forall t \in \mathbf{R}^{+}, \forall f \in L^{p}(M),
$$

for some $p, q$, and $\alpha$ satisfying $1 \leq p<q \leq \infty$, and $\alpha=1 / p-1 / q$. Then

$$
\left\|T_{t} f\right\|_{\infty} \leq C_{p, q} \phi(t)^{-1}\|f\|_{1} \quad \forall t \in \mathbf{R}^{+}, \forall f \in L^{1}(M) .
$$

Proof. Suppose that $1 \leq p<q \leq \infty$, and that

$$
\left\|T_{t} f\right\|_{q} \leq \phi(t)^{-\alpha}\|f\|_{p} \quad \forall f \in L^{p}(M), \forall t \in \mathbf{R}^{+} .
$$

For $T$ in $\mathbf{R}^{+}$and $f$ in $L^{1}(M) \cap L^{q}(M)$, denote by $K(T, f)$ the real number

$$
\sup _{0<t<T} \frac{\phi(t)^{1 / q^{\prime}}\left\|T_{t} f\right\|_{q}}{\|f\|_{1}} .
$$

Define $\theta$ in $[0,1]$ by the condition $1 / p=\theta / 1+(1-\theta) / q$, from which it follows that $\alpha=1 / p-1 / q=\theta / q^{\prime}$. By Hölder's inequality,

$$
\left\|T_{t} f\right\|_{p} \leq\left\|T_{t} f\right\|_{1}^{\theta}\left\|T_{t} f\right\|_{q}^{1-\theta} \leq\|\| T_{t}\|\|_{1}^{\theta}\|f\|_{1}^{\theta}\left\|T_{t} f\right\|_{q}^{1-\theta},
$$

so if $0<t<T$,

$$
\begin{aligned}
\left\|T_{2 t} f\right\|_{q} & \leq \phi(t)^{-\alpha}\left\|T_{t} f\right\|_{p} \\
& \leq \phi(t)^{-\alpha}\left\|\mid T_{t}\right\|\left\|_{1}^{\theta}\right\| f\left\|_{1}^{\theta}\right\| T_{t} f \|_{q}^{1-\theta} \\
& \leq \phi(t)^{-\alpha} \mid\left\|T_{t}\right\|\left\|_{1}^{\theta}\right\| f \|_{1}^{\theta}\left[\phi(t)^{-1 / q^{\prime}}\|f\|_{1} K(T, f)\right]^{1-\theta} \\
& =\phi(t)^{-1 / q^{\prime}}\left\|T_{t}\right\|\left\|_{1}^{\theta} K(T, f)^{1-\theta}\right\| f \|_{1},
\end{aligned}
$$

whence

$$
\begin{aligned}
K(T, f) & \leq K(2 T, f)=\sup _{0<t<T} \frac{\phi(2 t)^{1 / q^{\prime}}\left\|T_{2 t} f\right\|_{q}}{\|f\|_{1}} \\
& \leq \sup _{0<t<T}\left[\frac{\phi(2 t)}{\phi(t)}\right]^{1 / q^{\prime}}\left\|T_{t}\right\| \|_{1}^{\theta} K(T, f)^{1-\theta}
\end{aligned}
$$

and so

$$
K(T, f) \leq \sup _{0<t<T}\left[\frac{\phi(2 t)}{\phi(t)}\right]^{1 /\left(\theta q^{\prime}\right)}\left\|T_{t}\right\|_{1} \leq C_{\phi}^{1 /\left(\theta q^{\prime}\right)} C_{1} .
$$

The right-hand side of this last expression is independent of $f$ in $L^{1}(M) \cap$ $L^{q}(M)$ and $T$ in $\mathbf{R}^{+}$, so it follows that

$$
\left\|T_{t} f\right\|_{q} \leq C_{\dot{\phi}}^{1 /\left(\theta q^{\prime}\right)} C_{1} \phi(t)^{-1 / q^{\prime}}\|f\|_{1} \quad \forall \in L^{1}(M), \forall t \in \mathbf{R}^{+} .
$$

Since also

$$
\left\|T_{t} f\right\|_{1} \leq C_{1}\|f\|_{1} \quad \forall f \in L^{1}(M), \forall t \in \mathbf{R}^{+}
$$

by definition, we obtain by interpolation the estimate that

$$
\left\|T_{t} f\right\|_{p} \leq C_{\phi}^{(1-\theta) /\left(\theta q^{\prime}\right)} C_{1} \phi(t)^{-(1-\theta) / q^{\prime}}\|f\|_{1} \quad \forall f \in L^{1}(M), \forall t \in \mathbf{R}^{+} .
$$

Moreover, by duality, we observe that $T_{t}^{*}$ maps $L^{q^{\prime}}(M)$ to $L^{p^{\prime}}(M)$, whence by the same argument, $T_{t}^{*}$ maps $L^{1}(M)$ to $L^{p^{\prime}}(M)$, and again by duality $T_{t}$ maps $L^{p}(M)$ to $L^{\infty}(M)$; the corresponding estimate is

$$
\left\|T_{t} f\right\|_{\infty} \leq C_{\phi}^{1 /(\theta p)} C_{\infty} \phi(t)^{-1 / p}\|f\|_{p} \quad \forall \in L^{p}(M), \forall t \in \mathbf{R}^{+} .
$$


Combining the last two estimates, we find that

$$
\begin{aligned}
\left\|T_{2 t} f\right\|_{\infty} & \leq C_{\phi}^{1 /(\theta p)} C_{\infty} \phi(t)^{-1 / p}\left\|T_{t} f\right\|_{p} \\
& \leq C_{\phi}^{1 /(\theta p)+(1-\theta) /\left(\theta q^{\prime}\right)} C_{1} C_{\infty} \phi(t)^{-(1-\theta) / q^{\prime}-1 / p}\|f\|_{1} \\
& =C_{\phi}^{1 / \theta} C_{1} C_{\infty} \phi(t)^{-1}\|f\|_{1} \quad \forall f \in L^{1}(M), \forall t \in \mathbf{R}^{+},
\end{aligned}
$$

whence

$$
\begin{aligned}
\left\|T_{t} f\right\|_{\infty} & \leq C_{\phi}^{1 / \theta} C_{1} C_{\infty}\left[\frac{\phi(t)}{\phi(t / 2)}\right] \phi(t)^{-1}\|f\|_{1} \\
& \leq C_{\phi}^{1+1 / \theta} C_{1} C_{\infty} \phi(t)^{-1}\|f\|_{1} \quad \forall f \in L^{1}(M), \forall t \in \mathbf{R}^{+}
\end{aligned}
$$

i.e.,

$$
\left\|T_{t} f\right\|_{\infty} \leq C_{\phi}^{1+(1-1 / q) /(1 / p-1 / q)} C_{1} C_{\infty} \phi(t)^{-1}\|f\|_{1} \quad \forall f \in L^{1}(M), \forall t \in \mathbf{R}^{+} .
$$

Another duality argument shows this inequality also holds if $C_{\phi}^{1+(1-1 / q) /(1 / p-1 / q)}$ is replaced by $C_{\phi}^{1+(1 / p) /(1 / p-1 / q)}$, so we deduce that

$$
\left\|T_{t} f\right\|_{\infty} \leq C \phi(t)^{-1}\|f\|_{1} \quad \forall f \in L^{1}(M), \forall t \in \mathbf{R}^{+},
$$

where

$$
C=C_{\phi}^{1+\min \left\{1 / p, 1 / q^{\prime}\right\} /(1 / p-1 / q)} C_{1} C_{\infty}
$$

as claimed.

Lemma 1.4 is an extension of a technique of Davies [5], used in the proof of Theorem 2.4.2. Presumably this technique may also be combined with that of the proof of the Marcinkiewicz interpolation theorem (breaking up $f$ into two parts) to prove directly a strong type result.

Lemma 1.4. Suppose that $\left(T_{t}\right)_{t>0}$ is a semigroup satisfying the conditions

$$
\left\|T_{t} f\right\|_{p} \leq C\|f\|_{p} \quad \forall t \in \mathbf{R}^{+}, \forall f \in L^{p}(M)
$$

and

$$
\left\|T_{t} f\right\|_{q} \leq C \phi(t)^{-\alpha}\|f\|_{p} \quad \forall t \in \mathbf{R}^{+}, \forall f \in L^{p}(M),
$$

where $\alpha=1 / p-1 / q$. Then if $p<r<q$ and $\beta=1 / p-1 / r, \psi(\mathscr{G})^{-\beta}$ is of weak type $(p, r)$, and

$$
\left\|\psi(\mathscr{G})^{-\beta} f\right\|_{r, \infty} \leq C_{p, q, r}\|f\|_{p} \quad \forall f \in L^{p}(M) .
$$

Proof. First, observe that $\psi$ vanishes at most polynomially at 0 . Indeed, the doubling condition (b) on $\phi$ implies that $\phi$ grows at most polynomially at infinity, and

$$
\left|\psi(z)^{-\beta}\right| \leq C\left|\psi_{\beta}(z)\right| \leq C \int_{0}^{\infty} e^{-z t} \phi(t)^{\beta} \frac{d t}{t} \quad \forall z \in \mathbf{R}^{+} .
$$

Therefore, if $f$ is in $L^{p}(M) \cap L^{2}(M)$ and $k$ is large enough, $\left(I-T_{t}\right)^{k} f$ lies in $\operatorname{Dom}\left(\psi(\mathscr{G})^{-\beta}\right)$ for all $t$ in $\mathbf{R}^{+}$. Also, as $t$ tends to $\infty,\left(I-T_{t}\right)^{k} f$ tends to $f$, so that $L^{p}(M) \cap \operatorname{Dom}\left(\psi(\mathscr{G})^{-\beta}\right)$ is dense in $L^{p}(M)$.

Take $f$ in $L^{p}(M) \cap \operatorname{Dom}\left(\psi(\mathscr{G})^{-\beta}\right)$ and $\lambda$ in $\mathbf{R}^{+}$. We need to estimate the $L^{r, \infty}(M)$-norm of $\psi(\mathscr{G})^{-\beta} f$. In view of the general multiplier theorem 
(formula (0.4)), it will suffice to estimate the $L^{r, \infty}(M)$-norm of $\psi_{\beta}(\mathscr{G}) f$; i.e., we need to control the measure of the set $\left\{x \in M:\left|\psi_{\beta}(\mathscr{G}) f(x)\right|>\lambda\right\}$. To do this, take $s$ in $\mathbf{R}^{+}$, to be specified later. By spectral theory,

$$
\begin{aligned}
\psi_{\beta}(\mathscr{G}) f & =\int_{0}^{\infty} \phi_{\beta}(t) T_{t} f \frac{d t}{t}=\int_{0}^{s} \phi_{\beta}(t) T_{t} f \frac{d t}{t}+\int_{s}^{\infty} \phi_{\beta}(t) T_{t} f \frac{d t}{t} \\
& =B_{\beta, s} f+D_{\beta, s} f,
\end{aligned}
$$

say. Clearly

$$
\begin{aligned}
\left\|B_{\beta, s} f\right\|_{p} & \leq \int_{0}^{s} \phi_{\beta}(t)\left\|T_{t} f\right\|_{p} \frac{d t}{t} \\
& \leq C \int_{0}^{s} \phi(t)^{\beta}\|f\|_{p} \frac{d t}{t} \leq C \phi(s)^{\beta}\|f\|_{p},
\end{aligned}
$$

while

$$
\begin{aligned}
\left\|D_{\beta, s} f\right\|_{q} & \leq \int_{s}^{\infty} \phi_{\beta}(t)\left\|T_{t} f\right\|_{q} \frac{d t}{t} \leq C \int_{s}^{\infty} \phi(t)^{\beta-\alpha}\|f\|_{p} \frac{d t}{t} \\
& \leq C \phi(s)^{\beta-\alpha}\|f\|_{p} .
\end{aligned}
$$

Now

$$
\begin{aligned}
\left\{x \in M:\left|\psi_{\beta}(\mathscr{G}) f(x)\right|>\lambda\right\} & \subseteq\left\{x \in M:\left|B_{\beta, s} f(x)\right|>\frac{\lambda}{2}\right\} \\
\cup\left\{x \in M:\left|D_{\beta, s} f(x)\right|>\frac{\lambda}{2}\right\}, &
\end{aligned}
$$

and so, if $s$ is chosen so that $\phi(s)=\left(\|f\|_{p} / \lambda\right)^{(q-p) /(\beta p-\beta q+\alpha q)}$,

$$
\begin{aligned}
\mid\{x & \left.\in M:\left|\psi_{\beta}(\mathscr{G}) f(x)\right|>\lambda\right\} \mid \leq\left(\frac{\left\|B_{\beta, s} f\right\|_{p}}{\lambda / 2}\right)^{p}+\left(\frac{\left\|D_{\beta, s} f\right\|_{q}}{\lambda / 2}\right)^{q} \\
& \leq\left(\frac{2 C \phi(s)^{\beta}\|f\|_{p}}{\lambda}\right)^{p}+\left(\frac{2 C \phi(s)^{\beta-\alpha}\|f\|_{p}}{\lambda}\right)^{q} \\
& =C_{p, q, r}\left(\frac{\|f\|_{p}}{\lambda}\right)^{r}
\end{aligned}
$$

as required.

Lemma 1.5. Suppose that $\left(T_{t}\right)_{t>0}$ is a symmetric contraction semigroup and that

$$
\left\|\psi(\mathscr{G})^{-\alpha} f\right\|_{q, \infty} \leq C\|f\|_{p} \quad \forall f \in L^{p}(M)
$$

for some $p, q$ and $\alpha$ satisfying $1 \leq p<q \leq \infty$, and $\alpha=1 / p-1 / q<1$. Then if $p<r<q$ and $\beta=1 / p-1 / r$,

$$
\left\|T_{t} f\right\|_{r} \leq A_{p, q, r} \phi(t)^{-\beta}\|f\|_{p} \quad \forall t \in \mathbf{R}^{+}, \forall f \in L^{p}(M) .
$$

Proof. We suppose that $p>1$. By spectral theory, we may write (for $f$ in $\left.L^{p}(M) \cap L^{2}(M)\right)$,

$$
T_{t} f=\psi(\mathscr{G})^{-\alpha}\left[\psi(\mathscr{G})^{\alpha} e^{-t \mathscr{G}}\right] f
$$

by the result of Cowling [3] described in the introduction and the hypothesis of the lemma,

$$
\left\|T_{t} f\right\|_{q, \infty} \leq C\left\|\psi(\mathscr{G})^{\alpha} e^{-t \mathscr{G}} f\right\|_{p} \leq C \sup _{z \in \Gamma_{\omega}}\left|\psi(z)^{\alpha} e^{-t z}\right|\|f\|_{p}
$$


for some $\omega$ between 0 and $\pi / 2$. The hypothesis that the pair $(\phi, \psi)$ be regular implies that

$$
\sup _{z \in \Gamma_{\omega}}\left|\psi(z)^{\alpha} e^{-t z}\right| \leq C \sup _{z \in \mathbf{R}^{+}}\left|\psi(z)^{\alpha} e^{-t z}\right|,
$$

so if we can establish that

$$
\left|\psi(z)^{\alpha} e^{-t z}\right| \leq \phi(t)^{-\alpha} \quad \forall t \in \mathbf{R}^{+}, \forall z \in \mathbf{R}^{+},
$$

then it will follow that $\left\|T_{t} f\right\|_{q, \infty} \leq C \phi(t)^{-\alpha}\|f\|_{p}$ for all $f$ in $L^{p}(M)$ and all $t$ in $\mathbf{R}^{+}$. Real interpolation with the estimate $\left\|T_{t} f\right\|_{p} \leq\|f\|_{p}$ will then show that if $p \leq r<q$ and $\beta=1 / p-1 / r$,

$$
\left\|T_{t} f\right\|_{r} \leq A_{p, q, r} \phi(t)^{-\beta}\|f\|_{p} \quad \forall t \in \mathbf{R}^{+}, \forall f \in L^{p}(M),
$$

as required.

To establish (1.1), recall that $\psi_{\alpha}(z)=\int_{0}^{\infty} e^{-z s} \phi_{\alpha}(t) s^{-1} d s$ for all $z$ in $\mathbf{R}^{+}$, whence

$$
\begin{aligned}
\psi(z)^{\alpha} & \geq C \int_{0}^{\infty} e^{-z s} \phi(s)^{\alpha} \frac{d s}{s} \geq C \int_{t / 2}^{t} e^{-z s} \phi(s)^{\alpha} \frac{d s}{s} \\
& \geq C e^{-z t} \phi(t / 2)^{\alpha} \int_{t / 2}^{t} \frac{d s}{s} \geq C e^{-z t} \phi(t)^{\alpha} \quad \forall t \in \mathbf{R}^{+}, \forall z \in \mathbf{R}^{+},
\end{aligned}
$$

by properties of regularly related functions, and the desired inequality follows.

If $p=1$, then $1<q<\infty$; we write $T_{t} f=\left[\psi(\mathscr{G})^{\alpha} e^{-t \mathscr{G}}\right] \psi(\mathscr{G})^{-\alpha} f$, and observe that $\psi(\mathscr{G})^{-\alpha} f$ lies in $L^{q, \infty}(M)$, on which space $\psi(\mathscr{G})^{\alpha} e^{-t \mathscr{G}}$ acts boundedly. A simple modification of the proof above will treat this case.

This finishes the proof of Theorem 1.2.

It is interesting to compare Theorem 1.2 with Theorem 2.2 of $\mathrm{L}$. Hörmander [8]. Both theorems assert that if certain operators are of strong type $(p, q)$ (or of weak type $(p, q))$ for certain values of $p$ and $q$, then they are automatically of strong type $(p, q)$ for a range of $p$ 's and $q$ 's, and of weak type $(1, r)$ at the end of the range of these $p$ 's and $q$ 's.

We now present some consequences of Theorem 1.2.

Corollary 1.5. Suppose that $\left(e^{-t \mathscr{G}}\right)_{t>0}$ is a symmetric contraction semigroup, and that $(\phi, \psi)$ is a regularly related pair. If $\theta$ is in $(0,1)$, and $\tilde{\phi}=\phi^{1 / \theta}$, then the subordinated semigroup $\left(e^{-t \mathscr{G}^{\theta}}\right)_{t>0}$ is $\tilde{\phi}$-ultracontractive if and only if $\left(e^{-t \mathscr{G}}\right)_{t>0}$ is $\phi$-ultracontractive.

Proof. This is an immediate consequence of Theorem 1.2. For details of subordination, see, e.g., the book of K. Yosida [17].

It is not clear whether this result is true for general ultracontractive semigroups.

We shall not state our other corollary formally, but merely remark that for semigroups which satisfy estimates of the form

$$
\left\|T_{t} f\right\|_{\infty} \leq C_{0} t^{-\mu}\|f\|_{1} \quad \forall t \in(0,1], \forall f \in L^{1}(M)
$$

and

$$
\left\|T_{t} f\right\|_{\infty} \leq C_{1} t^{-\nu}\|f\|_{1} \quad \forall t \in[1, \infty), \forall f \in L^{1}(M)
$$


where $\mu$ and $\nu$ are positive, we obtain results very similar to those of Coulhon [2], by applying Theorem 1.2 in the particular case where $(\phi, \psi)$ is the pair of Lemma 1.1(a). Interesting examples of such semigroups were recently found by L. Saloff-Coste [12].

\section{UlTRACONTRACTIVITY AND "MULTIPLIER OPERATORS"}

Here again, $\mathscr{G}$ is the generator of a $\phi$-ultracontractive symmetric contraction semigroup $\left(T_{t}\right)_{t>0}$, and $(\phi, \psi)$ is a pair of regularly related functions, as defined in $\S 1$. Our concern is to find conditions on the function $m$ in order that the operator $m(\mathscr{G})$ extends to a bounded operator from $L^{p}(M)$ to $L^{q}(M)$ for suitable $p$ and $q$. The case where $p=q$ (in which $m$ is taken to be bounded) has already been treated. The first general result in this direction was obtained by Stein [13, Corollary 3, p. 121] and then refined and generalized by the firstnamed author [3, Theorem 3]. Our treatment here is based on the techniques employed in the second-named author's paper [9]. In particular, our first result, Theorem 2.1, is from [9], though the proof we give here is a little different.

Given a positive integer $N$, we denote by $m_{N}(t, \lambda)$ the function defined by the rule

$$
m_{N}(t, \lambda)=(t \lambda)^{N} e^{-t \lambda / 2} m(\lambda) \quad \forall t \in \mathbf{R}^{+}, \forall \lambda \in \mathbf{R}^{+},
$$

and by $\mathscr{M}_{\tau} m_{N}$ the Mellin $\tau$-transform of $m_{N}$ with respect to the second variable, i.e.,

$$
\left[\mathscr{M}_{\tau} m_{N}\right](t, u)=\int_{0}^{\infty} \lambda^{\tau-i u} m_{N}(t, \lambda) \frac{d \lambda}{\lambda} \quad \forall t \in \mathbf{R}^{+}, \forall u \in \mathbf{R} .
$$

In particular, ve write $\mathscr{M} m_{N}$ for the Mellin transform of $m_{N}$, thus:

$$
\left[\mathscr{M} m_{N}\right](t, u)=\int_{0}^{\infty}(t \lambda)^{N} e^{-t \lambda / 2} m(\lambda) \lambda^{-i u} \frac{d \lambda}{\lambda} \quad \forall t \in \mathbf{R}^{+}, \forall u \in \mathbf{R} .
$$

Theorem 2.1. Suppose that $m$ is a Borel-measurable function on $\mathbf{R}^{+}$such that for some positive integer $N$,

$$
\int_{-\infty}^{\infty} \sup _{t>0}\left|\left[\mathscr{M}_{\left.m_{N}\right]}\right](t, u)\right|\left\|\left|\mathscr{G}^{i u}\right|\right\|_{p} d u<\infty
$$

Then $m(\mathscr{G})$ is bounded on $L^{p}(M)$. Similarly, if

$$
\int_{-\infty}^{\infty} \sup _{t>0}\left|\left[\mathscr{M} m_{N}\right](t, u)\right|||\left|\mathscr{G}^{i u}\right| \|_{p, \infty} d u<\infty
$$

then $m(\mathscr{G})$ is bounded on $L^{p, \infty}(M)$.

Proof. Define functions $c: \mathbf{R} \rightarrow \mathbf{R}$ and $a_{u}: \mathbf{R}^{+} \rightarrow \mathbf{R}$ (where $u$ is in $\mathbf{R}$ ) by the formulae $c(u)=\sup _{t>0}\left|\left[\mathscr{M} m_{N}\right](t, u)\right|$, for all $u$ in $\mathbf{R}$, and $a_{u}(t)=$ $\left[\mathscr{M} m_{N}\right](t, u) / c(u)$, for all $t$ in $\mathbf{R}^{+}$, so that $\left\|a_{u}\right\|_{\infty} \leq 1$. Now

$$
\begin{aligned}
m(\lambda) & =\frac{1}{\Gamma(N+1)} \int_{0}^{\infty}(t \lambda)^{N+1} e^{-t \lambda} m(\lambda) \frac{d t}{t} \\
& =\frac{1}{2 \pi \Gamma(N+1)} \int_{0}^{\infty} \int_{-\infty}^{\infty}\left[\mathscr{M} m_{N}\right](t, u) \lambda^{i u} d u t \lambda e^{-t \lambda / 2} \frac{d t}{t} \\
& =\frac{1}{2 \pi \Gamma(N+1)} \int_{-\infty}^{\infty} \int_{0}^{\infty} \frac{\left[\mathscr{M} m_{N}\right](t, u)}{c(u)} t \lambda e^{-t \lambda / 2} \frac{d t}{t} c(u) \lambda^{i u} d u \\
& =\frac{1}{2 \pi \Gamma(N+1)} \int_{-\infty}^{\infty} \int_{0}^{\infty} a_{u}(t) t \lambda e^{-t \lambda / 2} \frac{d t}{t} c(u) \lambda^{i u} d u,
\end{aligned}
$$


provided the change of order of integration is justified. For any $\lambda$ in $\mathbf{R}^{+}$,

$$
\begin{aligned}
\int_{-\infty}^{\infty} & \int_{0}^{\infty}\left|a_{u}(t) t \lambda e^{-t \lambda / 2}\right| \frac{d t}{t}\left|c(u) \lambda^{i u}\right| d u \\
& \leq 2 \int_{-\infty}^{\infty}|c(u)| d u \leq 2 \int_{-\infty}^{\infty}|c(u)|||\left|\mathscr{G}^{i u}\right| \|_{p} d u<\infty
\end{aligned}
$$

so the interchange is legitimate. Note that $A_{u}: \lambda \mapsto \int_{0}^{\infty} a_{u}(t) t \lambda e^{-t \lambda / 2} d t / t$ is of Laplace transform type. In particular, it extends to a holomorphic function in the right half-plane, and for $\lambda$ in $\Gamma_{\pi / 2-\varepsilon}$,

$$
\begin{aligned}
\left|A_{u}(\lambda)\right| & =\left|\int_{0}^{\infty} a_{u}(t) t \lambda e^{-t \lambda / 2} \frac{d t}{t}\right| \\
& \leq \int_{0}^{\infty} t|\lambda| e^{-(t|\lambda| \sin \varepsilon) / 2} \frac{d t}{t}=\frac{2}{\sin \varepsilon} .
\end{aligned}
$$

Consequently $\left\|A_{u}(\mathscr{G})\right\|_{p} \leq C_{p}$ for all $u$ in $\mathbf{R}$, by (0.3), so that if (2.1) holds,

$$
\int_{-\infty}^{\infty}\left\|\left|A_{u}(\mathscr{G})\right|\right\|_{p}|c(u)|\left\|\left|\mathscr{G}^{i u}\right|\right\|_{p} d u<\infty .
$$

Since $u \mapsto a_{u}(\lambda)$ and $u \mapsto \lambda^{i u}$ are continuous functions for each $\lambda$ in $\mathbf{R}^{+}$, $u \mapsto a_{u}(\mathscr{G})$ and $u \mapsto \mathscr{G}^{i u}$ are strong operator topology continuous functions, and we may write $m(\mathscr{G})$ as a convergent integral of operators

$$
m(\mathscr{G})=\frac{1}{2 \pi \Gamma(N+1)} \int_{-\infty}^{\infty} A_{u}(\mathscr{G}) c(u) \mathscr{G}^{i u} d u .
$$

The variation of the proof needed to treat the $L^{p, \infty}(M)$ case is trivial.

We now look for easily verifiable conditions on $m$ which ensure that (2.1) or (2.2) holds.

Corollary 2.2. Suppose that $A$ and $\delta$ are in $\mathbf{R}^{+}$, and that $\left\|\mathscr{G}^{i u}\right\| \|_{p} \leq C \exp (\delta|u|)$ for all $u$ in $\mathbf{R}$. If $m$ extends to a bounded holomorphic function $m_{\omega}$ in $\Gamma_{\omega}$, where $\omega>\delta$, then $m(\mathscr{G})$ extends to a bounded operator on $L^{p}(M)$, and \|\|$m(\mathscr{G})\left\|_{p} \leq C_{\omega, \delta} A\right\| m_{\delta} \|_{\infty}$.

Proof. This follows from Theorem 2.1 and the Paley-Wiener theorem for Mellin transforms.

Suppose that $\chi$ is a positive integer and that $\alpha$ is in $\mathbf{R}^{+}$. We say that $m$ satisfies a Hörmander condition of order $(\alpha, \chi)$ if there exists $C$ such that

$$
\sup _{R>0} \psi(R)^{\alpha} \int_{R}^{2 R}\left|\lambda^{j} m^{(j)}(\lambda)\right| \frac{d \lambda}{\lambda} \leq C \quad \forall j \in\{0,1, \ldots, \chi\} .
$$

The smallest constant $C$ for which this inequality holds is called the Hörmander $(\alpha, \chi)$-constant of $m$. Similar conditions were introduced in Hörmander [8]. Note that our condition implies that $\sup _{\lambda>0}\left|\psi(\lambda)^{\alpha} m(\lambda)\right| \leq 2 C$.

Corollary 2.3. Suppose that $m$ satisfies a Hörmander condition of order $(0, \chi)$, with Hörmander $(0, \chi)$-constant $B$. Fix $p$ in $(1, \infty)$, and $A$ and $\tau$ in $\mathbf{R}^{+}$. If $\left\|\mathscr{G}^{i u}\right\|_{p} \leq A(1+|u|)^{\tau}$ for all $u$ in $\mathbf{R}$, and $\chi>\tau+1$, then $m(\mathscr{G})$ extends to a bounded operator on $L^{p}(M)$, and $\|m(\mathscr{G})\| \|_{p} \leq C_{\tau} A B$. Similarly, if 
$\left\|\mathscr{G}^{i u}\right\| \|_{p, \infty} \leq A(1+|u|)^{\tau}$ for all $u$ in $\mathbf{R}$, and $\chi>\tau+1$, then $m(\mathscr{G})$ extends to a bounded operator on $L^{p, \infty}(M)$, and $\|m(\mathscr{G})\|_{p, \infty} \leq C_{\tau}^{\prime} A B$.

Proof. The strong type result was proved by Meda [9, Theorem 4], by showing that the hypotheses of Theorem 2.1 are satisfied. The other result is similar.

Now we prove our results for the case where $p<q$. To state these, it is convenient to have more notation. Given $p$ and $q$ satisfying the condition that $1 \leq p \leq q \leq \infty$, we define $d([p, q], 2)$ by the rule $d([p, q], 2)=$ $\min _{r \in[p, q]}|1 / r-1 / 2|$.

Corollary 2.4. Assume that $m$ extends to an analytic function in the cone $\Gamma_{\omega}$ and that $\sup _{\lambda \in \Gamma_{\omega}}\left|\psi(\lambda)^{\alpha} m(\lambda)\right|<\infty$ for some $\alpha$ in $(0,1)$. Then $m(\mathscr{G})$ extends to a bounded operator from $L^{p}(M)$ to $L^{q}(M)$, provided that $d([p, q], 2)<\omega / \pi$ and $\alpha=1 / q-1 / p$, and to an operator of weak type $(1, r)$ if $1<r<\infty$, $d([1, r], 2)<\omega / \pi$ and $\alpha=1-1 / r$.

Proof. We consider first the strong type result. Choose $r$ in $[p, q]$ such that $|1 / r-1 / 2|=d([p, q], 2)$, and define $\beta$ to be $1 / p-1 / r$ and $\gamma$ to be $1 / r-1 / q$. Then $m(\lambda)=\psi(\lambda)^{-\gamma}\left[\psi(\lambda)^{\alpha} m(\lambda)\right] \psi(\lambda)^{-\beta} \quad \forall \lambda \in \mathbf{R}^{+}$, so on the appropriate domain, $m(\mathscr{G})=\psi(\mathscr{G})^{-\gamma}\left[\psi(\mathscr{G})^{\alpha} m(\mathscr{G})\right] \psi(\mathscr{G})^{-\beta}$; the first factor $\left(\psi(\mathscr{G})^{-\beta}\right)$ is bounded from $L^{p}(M)$ to $L^{r}(M)$, the second $\left(\left[\psi(\mathscr{G})^{\alpha} m(\mathscr{G})\right]\right)$ is bounded on $L^{r}(M)$, by Corollary 2.2, and the third $\left(\psi(\mathscr{G})^{-\gamma}\right)$ is bounded from $L^{r}(M)$ to $L^{q}(M)$, as required. The weak type case is similar.

For the rest of this section, we assume that there exists a positive real number $\sigma$ such that

$$
\left\|\left|\mathscr{G}^{i u}\right|\right\|_{p} \leq C_{p}(1+|u|)^{\sigma|1 / p-1 / 2|} \quad \forall u \in \mathbf{R}
$$

and

$$
\| \mathscr{G}^{i u}||_{p, \infty} \leq C_{p}^{\prime}(1+|u|)^{\sigma|1 / p-1 / 2|} \quad \forall u \in \mathbf{R},
$$

whenever $1<p<\infty$. This condition is fulfilled in many concrete cases such as nilpotent Lie groups (where $\mathscr{G}$ is any sublaplacean) and compact manifolds (where $\mathscr{G}$ is any elliptic positive selfadjoint operator).

Corollary 2.5. Suppose that $m$ satisfies a Hörmander condition of order $(\alpha, \chi)$ where $0<\alpha<1$ and $\chi$ is an integer no less than $\sigma / 2+1$. Then $m(\mathscr{G})$ extends to a bounded operator from $L^{p}(M)$ to $L^{q}(M)$ if $1<p<q<\infty$ and $\alpha=1 / p-1 / q$, and to an operator of weak type $(1, r)$, if $1<r<\infty$ and $\alpha=1-1 / r$.

Proof. This proof is a straightforward modification of that of Corollary 2.4, using Corollary 2.3 rather than Corollary 2.2 to bound the "middle factor". It is perhaps worth noting explicitly that the analyticity of $\psi$ implies that its derivatives can be controlled-using Cauchy's integral formula for holomorphic functions, one sees that $\left|\lambda^{j} \partial^{j} \psi(\lambda) / \partial \lambda^{j}\right|$ is of comparable size to $\psi(\lambda)$, for any positive integer $j$.

Corollary 2.6. Suppose that $m$ satisfies the condition

$$
\sup _{\lambda \in \mathbf{R}^{+}}\left|\psi(\lambda)^{\alpha} \lambda^{j} m^{(j)}(\lambda)\right| \leq C \quad \forall j \in\{0,1, \ldots, \chi\},
$$


for some integer $\chi$ no less than $\sigma / 2+1$ and some $\alpha$ in $(0, \mu)$. Then $m(\mathscr{G})$ extends to an operator of strong type $(p, q)$ if $1<p<q<\infty$ and $\alpha=$ $1 / p-1 / q$, and to an operator of weak type $(1, r)$ if $1<r<\infty$ and $\alpha=1-1 / r$.

Proof. It is obvious that if $m$ satisfies the condition of the corollary, then it satisfies a Hörmander condition of order $(\alpha, \chi)$, so the result follows from Corollary 2.5 .

\section{ULTRACONTRACTIVITY AND MAXIMAL OPERATORS}

In this section, we deal with semigroups $\left(T_{t}\right)_{t>0}$ satisfying an estimate of the form

$$
\left\|T_{t} f\right\|_{\infty} \leq A t^{-\mu}\|f\|_{1} \quad \forall t \in \mathbf{R}^{+}, \forall f \in L^{1}(M)
$$

for some positive $\mu$. We call such semigroups $\mu$-ultracontractive.

It is probably worth pointing out explicitly that Varopoulos (and some other authors) use $\mu / 2$ rather than $\mu$. This is because the index which appears when one studies the heat equation on a Euclidean space $\mathbf{R}^{d}$ is $d / 2$. However, if one considers the Poisson semigroup on the same space, then the appropriate index is $d$, and this example indicates that there is no ideal notation. We prefer our notation as it leads to cleaner formulae.

Let $m$ be a Borel measurable function on $\mathbf{R}^{+}$; recall that, for every nonnegative real number $\tau$, we have defined the Mellin $\tau$-transform $\mathscr{M}_{\tau} m$ of $m$ by the rule

$$
\left[\mathscr{M}_{\tau} m\right](u)=\int_{0}^{\infty} \lambda^{\tau-i u} m(\lambda) \frac{d \lambda}{\lambda} \quad \forall u \in \mathbf{R},
$$

whenever the integral on the right is absolutely convergent. If $\mathscr{M}_{\tau} m$ is integrable with respect to the Lebesgue measure on the real line, the following inversion formula holds:

$$
m(\lambda)=(2 \pi)^{-1} \int_{-\infty}^{\infty}\left[\mathscr{M}_{\tau} m\right](u) \lambda^{i u-\tau} d u \quad \forall \lambda \in \mathbf{R}^{+} .
$$

Recall also that we have defined the maximal operator $m_{*, \alpha}(\mathscr{G})$ as follows:

$$
m_{*, \alpha}(\mathscr{G}) f=\sup _{t>0}\left|t^{\alpha} m(t \mathscr{G}) f\right|,
$$

where $\alpha$ denotes a nonnegative real number. It is not a priori clear that $m_{*, \alpha}(\mathscr{G})$ is a well-defined operator. In the next proposition we shall show that this is indeed the case, at least for a large class of functions $m$. Boundedness results for the maximal operator $m_{*, 0}(\mathscr{G})$ were obtained by the first-named author [3]. Consequently, we shall deal with the case where $\alpha>0$ only. The proof of Theorem 3.1 follows closely the spirit of the proof of Theorem 6 in [3].

Theorem 3.1. Let $\mathscr{G}$ be the generator of a $\mu$-ultracontractive symmetric contraction semigroup, so that $\mathscr{G}^{-\alpha}$ is of weak type $(1, r)$ and of strong type $(p, q)$, when $1<p<q<\infty, 1<r<\infty$, and $1-1 / r=1 / p-1 / q=\alpha / \mu$. If $m$ is $a$ Borel measurable function on $\mathbf{R}^{+}$whose Mellin $\alpha$-transform $\mathscr{M}_{\alpha} m$ satisfies the estimate

$$
\int_{-\infty}^{\infty}\left|\left[\mathscr{M}_{\alpha} m\right](u)\right|||\left|\mathscr{G}^{i u-\alpha}\right| \|_{1 ; r, \infty} d u<\infty
$$


then the maximal operator $m_{*, \alpha}(\mathscr{G})$ is of weak type $(1, r)$. Similarly, if

$$
\int_{-\infty}^{\infty}\left|\left[\mathscr{M}_{\alpha} m\right](u)\right|||\left|\mathscr{G}^{i u-\alpha}\right|||_{p ; q} d u<\infty,
$$

then $m_{*, \alpha}(\mathscr{G})$ is of strong type $(p, q)$.

Proof. We prove only the weak type result. The other is very similar.

By spectral theory and the Mellin inversion formula we have, at least formally, that

$$
m(t \mathscr{G}) f=(2 \pi)^{-1} \int_{-\infty}^{\infty}\left[\mathscr{M}_{\alpha} m\right](u)(t \mathscr{G})^{i u-\alpha} f d u .
$$

Therefore

$$
\left|t^{\alpha} m(t \mathscr{G}) f\right| \leq(2 \pi)^{-1} \int_{-\infty}^{\infty}\left|\left[\mathscr{M}_{\alpha} m\right](u)\right|\left|\mathscr{G}^{i u-\alpha} f\right| d u,
$$

whence

$$
m_{*, \alpha}(\mathscr{G}) f \leq(2 \pi)^{-1} \int_{-\infty}^{\infty}\left|\left[\mathscr{M}_{\alpha} m\right](u)\right|\left|\mathscr{G}^{i u-\alpha} f\right| d u
$$

and

$$
\begin{aligned}
\left\|m_{*, \alpha}(\mathscr{G}) f\right\|_{r, \infty} & \leq \frac{1}{2 \pi} \int_{-\infty}^{\infty}\left|\left[\mathscr{M}_{\alpha} m\right](u)\right|\left\|\mathscr{G}^{i u-\alpha} f\right\|_{r, \infty} d u \\
& \leq \frac{1}{2 \pi}\left(\int_{-\infty}^{\infty}\left|\left[\mathscr{M}_{\alpha} m\right](u)\right|\left\|\mathscr{G}^{i u-\alpha} \mid\right\|_{1 ; r, \infty} d u\right)\|f\|_{1} \\
& =C\|f\|_{1} .
\end{aligned}
$$

It is not hard to justify the formal steps above. Since $C$ is finite, the integral

$$
\int_{-\infty}^{\infty}\left|\left[\mathscr{M}_{\alpha} m\right](u)\right|\left|\mathscr{G}^{i u-\alpha} f\right| d u
$$

converges as a Bochner integral in the Banach space $L^{r, \infty}(M)$ as long as the map $u \mapsto \mathscr{G}^{i u-\alpha} f$ is measurable. Now, the map $u \mapsto \mathscr{G}^{i u}\left(\mathscr{G}^{-\alpha} f\right)$ is strong operator topology continuous on $L^{p}(M)$ for all $p$ in $(1, \infty)$, hence on $L^{r, \infty}(M)$. Thus, the function $u \mapsto \mathscr{G}^{i u}\left(\mathscr{G}^{-\alpha} f\right)$ is measurable and

$$
\int_{-\infty}^{\infty}\left[\mathscr{M}_{\alpha} m\right](u)(t \mathscr{G})^{i u}\left(\mathscr{G}^{-\alpha} f\right) d u
$$

converges as a $L^{r, \infty}(M)$-valued integral and the function of $t$ thus obtained is continuous. Finally, $m_{*, \alpha}(\mathscr{G}) f$ makes sense in the Banach lattice $L^{r, \infty}(M)$ by the continuity of the function $t \mapsto t^{\alpha} m(t \mathscr{G}) f$. This concludes the proof of the proposition.

We now discuss some consequences of Theorem 3.1.

Let $a^{(\delta)}(t \lambda)$ and $a^{(\delta)}(t \mathscr{G})$ be the analytic families of functions and corresponding operators defined, for all $\delta$ in $\Gamma$, by the rules

$$
a^{(\delta)}(t \lambda)=\frac{1}{\Gamma(\delta)} \int_{0}^{1}(1-s)^{\delta-1} \exp (-s t \lambda) d s \quad \forall \lambda \in \mathbf{R}^{+}
$$

and

$$
a^{(\delta)}(t \mathscr{G}) f=\frac{1}{\Gamma(\delta)} \int_{0}^{1}(1-s)^{\delta-1} T_{s t} f d s \quad \forall f \in L^{p}(M) .
$$


For every fixed positive $t$ the family $\left(a^{(\delta)}(t \mathscr{G})\right)_{\operatorname{Re} \delta>0}$ admits an analytic continuation to the whole complex plane (see Stein [13, p. 77]). In particular $a^{(0)}(t \mathscr{G})=T_{t}$ and, for every positive integer $k, a^{(-k)}(t \mathscr{G})=t^{k} d^{k} / d t^{k} T_{t}$. As an application of Theorem 3.1, we now investigate the behaviour of the maximal operators $a_{*, \alpha}^{(\delta)}(\mathscr{G})$ defined by the rule

$$
a_{*, \alpha}^{(\delta)}(\mathscr{G}) f=\sup _{t>0}\left|t^{\alpha} a^{(\delta)}(t \mathscr{G}) f\right| .
$$

The case where $\alpha=0$ was treated in Theorem 6 of Cowling [3], so we assume that $\alpha \neq 0$. An easy calculation shows that if $\operatorname{Re} \delta>0$ and $0<\operatorname{Re} \alpha<1$, the Mellin $\alpha$-transform of the function $a^{(\delta)}$ is given by

$$
\left[\mathscr{M}_{\alpha} a^{(\delta)}\right](u)=\frac{\Gamma(1-\alpha+i u) \Gamma(\alpha-i u)}{\Gamma(\delta+1-\alpha+i u)} \quad \forall u \in \mathbf{R} .
$$

Notice that by the asymptotics for the $\Gamma$-function, the integral

$$
\int_{-\infty}^{\infty}\left|\left[\mathscr{M}_{\alpha} a^{(\delta)}\right](u)\right|\left\|\left|\mathscr{G}^{i u}\right|\right\|_{p} d u
$$

is convergent for all $p$ in $(1, \infty)$, provided that $\alpha \neq 1$ or $\alpha=1$ and $\delta=0$.

Corollary 3.2. Assume that $\mathscr{G}$ is the generator of a $\mu$-ultracontractive symmetric contraction semigroup. Then if $0<\alpha<1$ and $\delta$ is in $\mathbf{C}$, the maximal operator $a_{*, \alpha}^{(\delta)}(\mathscr{G})$ is of strong type $(p, q)$ for all $p, q$ such that $1<p<q<\infty$ and $1 / p-1 / q=\alpha / \mu$, and it is of weak type $(1, r)$ when $1<r<\infty$ and $1-1 / r=$ $\alpha / \mu$.

Proof. Immediate from Theorem 3.1 and the above computations.

It is possible to formulate a more general version of this result; we do so, although we do not know any immediate applications. A further generalization replaces $\phi(t)^{\beta}$ with $h(t)$ and $\psi(\lambda)^{-\beta}$ with $k(\lambda)$, for other functions $h$ and $k$. The proof is almost the same as that of Theorem 3.1, and we omit it.

Theorem 3.3. Let $(\varphi, \psi)$ be a regularly related pair of functions, and let $\mathscr{G}$ be the generator of a $\phi$-ultracontractive symmetric contraction semigroup. Given a Borel measurable function $m$ on $\mathbf{R}^{+}$and a subinterval $\mathscr{T}$ of $\mathbf{R}^{+}$, define the function $m_{\beta}^{b}$ by the rule

$$
m_{\beta}^{b}(t, \lambda)=\phi(t)^{\beta} \psi(\lambda)^{\beta} m(t \lambda) \quad \forall t \in \mathscr{T}, \forall \lambda \in \mathbf{R}^{+} .
$$

If $1<r<\infty$ and $\beta=1-1 / r$, and

$$
\int_{-\infty}^{\infty} \sup _{t \in \mathscr{T}}\left|\left[\mathscr{M} m_{\beta}^{b}\right](t, u)\right|\left\||| \mathscr{G}^{i u} \psi(\mathscr{G})^{-\beta}(\mathscr{G}) \mid\right\|_{1 ; r, \infty} d u<\infty
$$

then the maximal operator

$$
f \mapsto \sup _{t \in \mathscr{T}}\left|\phi(t)^{\beta} m(t \mathscr{G}) f\right|
$$

is of weak type $(1, r)$. Similarly, if $1<p<q<\infty$ and $\beta=1 / p-1 / q$, and

$$
\int_{-\infty}^{\infty} \sup _{t>1}\left|\left[\mathscr{M} m_{\beta}^{b}\right](t, u)\right|\left\|\mid \mathscr{G}^{i u} \psi(\mathscr{G})^{-\beta}(\mathscr{G})\right\| \|_{p ; q} d u<\infty
$$

then the maximal operator $(3.1)$ is of strong type $(p, q)$. 
Other mixed norm estimates can be obtained by the methods developed in the present section: details and applications will be given elsewhere.

\section{FurTHER GENERALIZATIONS}

The aim of this section is to present some extensions of the theory developed in the first three, and a few comments. To motivate the generalizations, let us observe that there are many "natural" ultracontractive semigroups for which ||$\left|T_{t}\right| \|_{1 ; \infty}^{-1}$ behaves polynomially for small $t$ but not for large $t$. For example, heat diffusion on a compact manifold or on a hyperbolic manifold both give rise to semigroups such that, if $\phi(t)^{-1}=\|\| T_{t} \|_{1 ; \infty}$ for all $t$ in $\mathbf{R}^{+}$, then $\phi$ cannot be one of a regularly related pair of functions. One family of extensions arises from the remark after the enunciation of Theorem 1.2.

Given positive numbers $\alpha$ and $\varepsilon$ we consider the "Bessel potentials" $(\varepsilon+\mathscr{G})^{-\alpha}$. Notice that

$$
(\varepsilon+\mathscr{G})^{-\alpha}=\frac{1}{\Gamma(\alpha)} \int_{0}^{\infty} t^{\alpha} e^{-\varepsilon t} T_{t} \frac{d t}{t} .
$$

It is intuitively obvious that the behaviour of $(\varepsilon+\mathscr{G})^{-\alpha}$ is related to the behaviour of $T_{t}$ for small values of $t$, and the rest of our paper illustrates this.

The following result was also observed by Davies [5, Corollary 2.4.3].

Theorem 4.1. Fix $\mu$ in $\mathbf{R}^{+}$. For a symmetric contraction semigroup, the following conditions are equivalent:

(a)

$$
\left\|T_{t} f\right\|_{\infty} \leq A t^{-\mu}\|f\|_{1} \quad \forall t \in(0,1], \forall f \in L^{1}(M)
$$

(b) for all $r, \alpha$ and $\varepsilon$ such that $1<r<\infty, \alpha=\mu(1-1 / r)$, and $0<\varepsilon<\infty$,

$$
\left\|(\varepsilon+\mathscr{G})^{-\alpha} f\right\|_{r, \infty} \leq C_{r, \varepsilon}\|f\|_{1} \quad \forall f \in L^{1}(M) ;
$$

(c) for all $p, q, \alpha$ and $\varepsilon$ such that $1<p<q<\infty, \alpha=\mu(1 / p-1 / q)$, and $0<\varepsilon<\infty$,

$$
\left\|(\varepsilon+\mathscr{G})^{-\alpha} f\right\|_{q} \leq C_{p, q, \varepsilon}\|f\|_{p} \quad \forall f \in L^{p}(M) ;
$$

(d) there exist $p, q, \alpha$ and $\varepsilon$ such that $1<p<q<\infty, \alpha=\mu(1 / p-1 / q)$, $0<\varepsilon<\infty$, and

$$
\left\|(\varepsilon+\mathscr{G})^{-\alpha} f\right\|_{q} \leq C\|f\|_{p} \quad \forall f \in L^{p}(M) ;
$$

(e) there exist $p, q, \alpha$ and $\varepsilon$ such that $1<p<q<\infty, \alpha=\mu(1 / p-1 / q)$, $0<\varepsilon<\infty$, and

$$
\left\|(\varepsilon+\mathscr{G})^{-\alpha} f\right\|_{q, \infty} \leq C^{\prime}\|f\|_{p} \quad \forall f \in L^{p}(M) .
$$

Proof. In view of the fact that $(\varepsilon+\mathscr{G})$ generates the symmetric contraction semigroup $\left(e^{-\varepsilon t} T_{t}\right)_{t>0}$, to which Theorem 1.2 applies, with $\phi(t)=t^{\mu}$ for all $t$ in $\mathbf{R}^{+}$, this is already proved.

Note that if any of the equivalent condition of the above theorem is satisfied, and $\mu^{\prime}>\mu$, then $\left\|T_{t} f\right\|_{\infty} \leq A t^{-\mu^{\prime}}\|f\|_{1}$ for all $t$ in $(0,1]$ and all $f$ in $L^{1}(M)$. It follows that (b) holds if $\alpha \geq \mu(1-1 / r)$ and (c) holds if $\alpha \geq \mu(1 / p-1 / q)$. 
It should be quite obvious by now that multiplier results and maximal theorems for $\mu$-ultracontractive operators can be extended to semigroups satisfying (4.1). We give two more such results without proofs. It will not be hard for the interested reader to supply them by adapting the proofs and ideas above.

Here is a version of Corollary 2.4, and an extension of Theorem 3.1. We retain the notation therein.

Corollary 4.2. Fix $\mu$ in $\mathbf{R}^{+}$. Assume that $\left(T_{t}\right)_{t>0}$ is a symmetric contraction semigroup satisfying the estimate (4.1), that $m$ extends to an analytic function $m_{\omega}$ in the cone $\Gamma_{\omega}$, and that

$$
\sup _{\lambda \in \Gamma_{\omega}}\left|(1+\lambda)^{\alpha} m_{\omega}(\lambda)\right|<\infty
$$

for some $\alpha$ such that $0<\alpha<\mu$. Then $m(\mathscr{G})$ extends to a bounded operator from $L^{p}(M)$ to $L^{q}(M)$, provided that $1<p<q<\infty, d([p, q], 2)<\omega / \pi$ and $1 / p-1 / q \leq \alpha / \mu$, and to an operator of weak type $(1, r)$ if $1<r<\infty$, $d([1, r], 2)<\omega / \pi$ and $1-1 / r \leq \alpha / \mu$.

Theorem 4.3. Fix $\mu$ in $\mathbf{R}^{+}$. Let $\mathscr{G}$ be the generator of a symmetric contraction semigroup satisfying condition (4.1). Given a Borel measurable function $m$ on $\mathbf{R}^{+}$, define the function $m_{\alpha}^{\sharp}$ by the rule

$$
m_{\alpha}^{\sharp}(t, \lambda)=t^{\alpha}(1+\lambda)^{\alpha} m(t \lambda), \quad \forall t \in(0,1], \forall \lambda \in \mathbf{R}^{+}
$$

whenever $0<\alpha<\mu$. If $1<r<\infty, 1-1 / r=\alpha / \mu$, and

$$
\int_{-\infty}^{\infty} \sup _{0<t \leq 1}\left|\left[\mathscr{M} m_{\alpha}^{\sharp}\right](t, u)\right||| \mathscr{G}^{i u}(I+\mathscr{G})^{-\alpha} \mid \|_{1 ; r, \infty} d u<\infty,
$$

then the maximal operator

$$
f \mapsto \sup _{0<t \leq 1}\left|t^{\alpha} m(t \mathscr{G}) f\right|
$$

is of weak type $(1, r)$. Similarly, if

$$
\left.\int_{-\infty}^{\infty} \sup _{0<t \leq 1}\left|\left[\mathscr{M} m_{\alpha}^{\sharp}\right](t, u)\right||| \mathscr{G}^{i u}(I+\mathscr{G})^{-\alpha}||\right|_{p ; q} d u<\infty,
$$

and $1<p<q<\infty$ and $1 / p-1 / q=\alpha / \mu$, then the maximal operator (4.2) is of strong type $(p, q)$.

We conclude by observing that the following "parabolic Harnack inequality" holds. This result should be compared with Theorem $M$ of Varopoulos [15]. Note that we do not make any positivity assumption on the semigroup $\left(T_{t}\right)_{t>0}$ or "regularity assumptions" like those of Varopoulos [16].

Proposition 4.4. Assume that $\left(T_{t}\right)_{t>0}$ is a $\phi$-ultracontractive symmetric contraction semigroup, and that $f$ is in $L^{2}(M)$. Define $u: M \times \mathbf{R}^{+} \rightarrow \mathbf{C}$ by the rule $u(\cdot, t)=T_{t} f(\cdot)$ for every positive $t$. Then

$$
\|u(\cdot, 1)\|_{\infty} \leq C_{\mu}\left(\int_{1 / 2}^{1} \int_{M}|u(x, t)|^{2} d x d t\right)^{1 / 2} .
$$


Proof. Notice that for $t$ in $(0,1)$,

$$
\begin{aligned}
\|u(\cdot, 1)\|_{\infty}^{2} & =2 \int_{1 / 2}^{1}\|u(\cdot, 1)\|_{\infty}^{2} d t=2 \int_{1 / 2}^{1}\left\|T_{t} f\right\|_{\infty}^{2} d t \leq 2 \int_{1 / 2}^{1} \phi(t)^{-1}\left\|T_{t} f\right\|_{2}^{2} d t \\
& \leq 2 \phi(1 / 2)^{-1} \int_{1 / 2}^{1} \int_{M}\left|T_{t} f(x)\right|^{2} d x d t .
\end{aligned}
$$

The first of the above inequalities is an immediate consequence of the contractivity of $T_{t}$ on $L^{\infty}(M)$ and the second follows from Theorem 1.2.

\section{REFERENCES}

1. E. A. Carlen, S. Kusuoka, and D. W. Stroock, Upper bounds for symmetric Markov transition functions, Ann. Inst. H. Poincaré, Probabilité et Statisque, Suppl. au No. 2, 1987, pp. 245287.

2. T. Coulhon, Dimension à l'infini d'un semi-group analytique, Bull. Sci. Math. 114 (1990), 485-500.

3. M. G. Cowling, Harmonic analysis on semigroups, Ann. of Math. (2) 117 (1983), 267-283.

4. E. B. Davies, Hypercontractive and related bounds for double well Schrödinger operators, Quart. J. Math. Oxford Ser. (2) 34 (1983), 407-421.

5. __ Heat kernels and spectral theory, Cambridge Tracts in Math., no. 92, Cambridge Univ. Press, 1989.

6. E. B. Davies and B. Simon, Ultracontractivity and the heat kernel for Schrödinger operators and Dirichlet Laplacians, J. Funct. Anal. 59 (1984), 335-395.

7. L. Gross, Logarithmic Sobolev inequalities, Amer. J. Math. 97 (1976), 1061-1083.

8. L. Hörmander, Estimates for translation invariant operators in $L^{p}$ spaces, Acta Math. 104 (1960), 93-140.

9. S. Meda, A general multiplier theorem, Proc. Amer. Math. Soc. 110 (1990), 639-647.

10. J. Moser, A Harnack inequality for parabolic differential equations, Comm. Pure Appl. Math. 17 (1964), 101-134.

11. __ Correction to “A Harnack inequality for parabolic differential equations", Comm. Pure Appl. Math. 20 (1967), 231-236.

12. L. Saloff-Coste, Analyse sur les groupes de Lie à croissance polynomiale, Ark. Math. 28 (1990), 315-331.

13. E. M. Stein, Topics in harmonic analysis related to the Littlewood-Paley theory, Ann. of Math. Stud., no. 63, Princeton Univ. Press, Princeton, N. J., 1970.

14. E. M. Stein and G. Weiss, Introduction to Fourier analysis on Euclidean spaces, Princeton Univ. Press, Princeton, N. J., 1970.

15. N. T. Varopoulos, Isoperimetric inequalities and Markov chains, J. Funct. Anal. 63 (1985), 215-239.

16. __ Hardy-Littlewood theory for semigroups, J. Funct. Anal. 63 (1985), 240-260.

17. K. Yosida, Functional analysis, 4th ed., Springer-Verlag, Berlin, Heidelberg, and New York, 1974.

School of Mathematics, University of New South Wales, Kensington N. S. W. 2033, Australia

E-mail address: michaelc@hydra.maths.unsw.edu.au

Dipartimento di Matematica, Università di Trento, 38050 Povo (TN), Italia

Current address (S. Meda): Dipartimento di Matematica, Politecnico di Milano, Via Bonardi 9, 20133 Milano, Italia 